\title{
RESEARCH IN NUCLEAR ASTROPHYSICS: STELLAR COLLAPSE AND SUPERNOVAE
}

\author{
Performance Reports \\ USDOE Grant No. DE-FG02-87ER40317
}

\author{
J. M. Lattimer and A. Yahil \\ Dept. of Earth \& Space Sciences \\ State University of New York at Stony Brook
}

December 1, 1991 - November 30, 1992

\section{DISCLAIMER}

This report was prepared as an account of work sponsored by an agency of the United States Government. Neither the United States Government nor any agency thereof, nor any of their employees, makes any warranty, express or implied, or assumes any legal liability or responsibility for the accuracy, completeness, or usefulness of any information, apparatus, product, or process disclosed, or represents that its use would not infringe privately owned rights. Reference herein to any specific commercial product, process, or service by trade name, trademark, manufacturer, or otherwise does not necessarily constitute or imply its endorsement, recommendation, or favoring by the United States Government or any agency thereof. The views and opinions of authors expressed herein do not necessarily state or reflect those of the United States Government or any agency thereof. 
This progress report describes the nuclear astrophysics research activities in the Earth and Space Sciences Department at Stony Brook during the last year. Our research focused on three aspects of nuclear astrophysics: (1) the equation of state of hot, dense matter, (2) the origin of supernovae and neutron stars, (3) the early cooling epoch of neutron stars. The following contains detailed reports which summarize each completed project.

\section{Rapid Cooling of Neutron Stars by Hyperons and $\Delta$ Isobars*}

M. Prakash (Dept. of Physics), M. Prakash (Dept. of Physics), J. M. Lattimer and C. J. Pethick (Nordita)

We show that direct Urca processes with hyperons and/or nucleon isobars can occur in dense matter as long as the concentration of $\Lambda$ hyperons exceeds a critical value that is less than $3 \%$ and is typically about $0.1 \%$. The neutrino luminosities from the hyperon Urca processes are about 5-100 times less than the typical luminosity from the nucleon direct Urca process, if the latter process is not forbidden, but they are larger than those expected from other sources. These new direct Urca processes provide avenues for rapid cooling of neutron stars which invoke neither exotic states nor the large proton fraction (of order 0.11-0.15) required for the nucleon direct Urca process.

*Astrophys. J. Letters, 390, L77 (1992)

\section{A Generalized Equation of State For Hot, Dense Matter*}

\section{J. M. Lattimer and F. D. Swesty}

An equation of state for hot, dense matter is presented in a form that is sufficiently rapid to use directly in hydrodynamical simulations. It contains an adjustable nuclear force that accurately models both potential and mean-field interactions, and it allows for the input of various nuclear parameters, some of which are not yet experimentally well-determined. These include the bulk incompressibility parameter, the bulk and surface symmetry energies, the symmetric matter surface tension, and the nucleon effective masses. The equation of state is modelled after the Lattimer, Lamb, Pethick, and Ravenhall (1985), hereafter referred to as LLPR, compressible liquid droplet model for nuclei, and includes the effects of interactions and degeneracy of the nucleons outside nuclei. Account is also taken of nuclear deformations and the phase transitions from nuclei to uniform nuclear matter at subnuclear densities. Comparisons of this equation of state are made to the results of the LLPR model and the Cooperstein-Baron equation of state. The effects of varying the bulk incompressibility are also investigated.

Our approach in this paper is to follow the detailed calculations of LLPR, further simplifying the free energy function of the finite temperature compressible liquid drop model and thus the equilibrium equations derived from its minimization. We sought a form in which a lower order Newton-Raphson iteration is acheived (the original LLPR model is 5dimensional) and the results followed those of LLPR to a reasonable accuracy, for the same underlying nuclear force. However, our model contains the nuclear parameters previously mentioned as inputs which can be easily, and consistently, altered. It is also straightforward to substitute any other model for the nucleon-nucleon force provided that an expression for 
the energy as a function of density, temperature and composition is available. Inasmuch as the model presented here reproduced the original LLPR calculations, we have confidence that the detailed results for other choices of nuclear parameters or forces will also be faithfully represented. Our goal was to present our calculations as an algorithm that is sufficiently rapid and flexible to be useful in many astrophysical calculations.

${ }^{*}$ Nucl. Phys. A535, 331 (1991)

\section{Effects of the Equation of State in Neutron Stars and in Stellar Collapse*}

\section{J. M. Lattimer and A. Burrows (Arizona)}

Some effects of the equation of state of dense matter on the structure of neutron stars and on gravitational collapse supernovae are discussed. We discuss which nuclear parameters are most impo:tant for the equation of state, and constraints on them that have been obtained from litboratory experiments. We then discuss how neutron stars and supernovae depend upon and, therefore, could constrain, these parameters. Some structural constraints on neutron stars are available from observations, but these cannot, at present, set limits on important nuclear parameters such as the bulk incompressibility parameter or the bulk symmetry energy. However, the mechanism of Type II, or gravitational collapse, supernovae may depend on these nuclear parameters and upon other aspects of the equation of state. We examine the equation of state in the important regime just below nuclear density (about $2 \times 10^{14} \mathrm{~g} \mathrm{~cm}^{-3}$ ) in which nuclei become deformed and undergo possible phase transitions to other shapes such as an inside-out bubble phase, and a phase transition to uniform nuclear matter. The pressure and adiabatic index in this density regime is sensitive chiefly to the incompressibility parameter of bulk nuclear matter. The supernova mechanism will also depend upon the effective neutrino-nucleus coherent scattering rate, which in turn is sensitive to the nuclear size and to ion-ion correlations. Calculations of the coherent scattering rate are compared to those currently used in other stellar collapse simulations.

${ }^{*}$ in Proceedings of the ESO/EIPC Workshop on SN 1987A and Other Supernovae, ed. J. Danziger and K. Kjä̈ (European Southern Observatory, Garching bei Müichen), 69 (1991)

\section{Rapid Cooling and the Stucture of Neutron Stars*}

J. M. Lattimer, K. A. Van Riper (Los Alamos), M. Prakash and M. Prakash

Recently proposed neutrino emission processes in high-density matter result in the relatively rapid cooling of a neutron star's interior followed by a precipitous drop in the surface temperature. We show that the time interval between the formation of the neutron star and the drop in the surface temperature is primarily determined by the structure of the neutron star, and is relatively insensitive to the rapid cooling mechanism itself. Thus, obbservations of thermal emissions from neturon stars have the potential for constraining the neutron star's radius and the underlying equation of state of dense matter.

*manuscript in preparation 


\section{The Equation of State in Neutron Star Matter*}

J. M. Lattimer

The role of the equation of state of dense matter on the structure of neutron stars and on gravitational collapse supernovae are discussed. We discuss which nuclear parameters are most important for the equation of state, and constraints on them that have been obtained from laboratory experiments. We then discuss how neutron stars and supernovae depend upon and, therefore, could constrain, these parameters. Some structural constraints on neutron stars are available from observations, but these cannot, at present, set limits on important nuclear parameters such as the bulk incompressibility parameter or the bulk symmetry energy. However, the mechanism of Type II, or gravitational collapse, supernovae may depend on these nuclear parameters and upon other aspects of the equation of state. We examine the equation of state in the important regime just below nuclear density (about $2 \times 10^{14} \mathrm{~g} \mathrm{~cm}^{-3}$ ) in which nuclei become deformed and undergo possible phase transitions to other shapes such as an inside-out bubble phase, and a phase transition to uniform nuclear matter. The pressure and adiabatic index in this density regime is sensitive chiefly to the incompressibility parameter of bulk nuclear matter. The supernova mechanism will also depend upon the effective neutrino-nucleus coherent scattering rate, which in turn is sensitive to the nuclear size and to ion-ion correlations. Calculations of the coherent scattering rate are compared to those currently used in other stellar collapse simulations. The implications of altering the neutrino opacities on neutron star cooling is graphically demonstrated.

*in Proceedings of the U.S.-Japan Joint Seminar on the Structure and Evolution of Neutron Stars, eds. R. Tamagaki and S. Tsuruta (Addison-Wesley, New York), in press (1992).

\section{The Role of the Equation of State in the "Prompt" Phase of Core Collapse Supernovae*}

F.D. Swesty, J. M. Lattimer, and E. S. Myra (Intel Corp.)

Although a number of studies have been undertaken in an attempt to ascertain the role of the equation of state (EOS) in the initial phases of supernovae, none of these studies have employed EOS's that are consistently parameterized in terms of the nuclear force parameters. We have recently developed [1] a compressible liquid drop model EOS that is based on the LLPR [2] liquid droplet model EOS and that is consistently parameterized. We have combined this EOS with our radiation hydrodynamic simulation code in order to attempt to delineate the role of the EOS in the "prompt" phase. However, one cannot simply vary the EOS nuclear force parameters independently and expect to achieve a physically realistic EOS, since correlations exist between these parameters because of nuclear systematics. This is particularly true for the case of nuclear symmetry energies, since the volume and surface symmetry energies are strongly correlated [3]. Also, the nuclear specific heat and the incompressibility are strongly linked. Another important constraint [4] is the observed neutron star mass of $1.44 \mathrm{M}_{\odot}$ for the binary pulsar system PSR1913+16. Any EOS applied to models of supernovae must be able to build a neutron star of at least $1.44 \mathrm{M}_{\odot}$. Lastly, the EOS must remain causal at all densities encountered in the neutron star or supernova models. We have conducted radiation hydrodynamic simulations with 
consistently varied nuclear force parameters that allow the EOS to meet all of these constraints. We find that the role of these parameters in the supernova is surprisingly different than what is commonly thought. Ostensibly, the nuclear incompressibility and symmetry energy strongly affect the shock dynamics. Our initial results indicate that when the compression is constrained by the PSR1913+16 limit, and the symmetry energy is realistically constrained, the shock dynamics is unaffected by variations in these parameters. In contrast, the symmetry energies may have far more substantial role in determining both the neutrino signature and the magnitude of the convective instabilities.

*manuscript in preparation

[1] Lattimer, J. M. and Swesty, F. D., 1991, Astrophys. J. A535, 331.

[2] Lattimer, J. M., Pethick, C. J., Ravenhall, D. G., and Lamb, D. Q., 1985, Nucl. Phys. A432, 646.

[3] Lattimer, J. M., 1992, In The Structure and Evolution of Neutron Stars, eds. R. Tamagaki and S. Tsuruta (Addison-Wesley, New York), in press.

[4] Van Riper, K. A. 1979, Ap. J., 232, 558.

\section{Rapid Cooling and Structure of Neutron Stars*}

K. A. Van Riper and J. M. Lattimer

A neutron star cooling very rapidly in the center will undergo a sharp decrease in surface temperature characterized by the timescale for thermal diffusion through the crust. This time depends on the square of the thickness of the crust, and is also influenced by the details of the neutron superfluidity in the crust. An observational determination of this time will limit the radius of the star, and, hence, the mass and high density equation of state. For stars cooling by the direct Urca process, the surface temperature in the epoch between the sharp drop and the age of about $10^{5}$ years depends strongly on the details of the superfluid gaps above nuclear density and on the central density of the star. The parameters of the high density gaps are uncertain, as is the central density. It is also possible that higher order gaps exist for both the neutron and protons; these gaps will also influence the surface temperature during this epoch.

*in Proceedings of the Taos Meeting on Neutron Stars and Gamma Ray Bursts, ed. K. A. Van Riper.

\section{Neutrino Transport in Supernovae*}

J. A. Miralles, J. M. Ibáñez, J. M. Marti (Valencia), and A. Pérez (Valencia),

Several methods which have been proposer the last few years for treating neutrino transport in stellar collapse problems are analyzed.

*Astrophysics Letters and Communications, 28, 223 (1992) 


\title{
9. The Boltzmann Equation in General Relativistic Rotating Systems: Cool- ing of Rotating Neutron Stars*
}

\author{
J. A. Miralles, K. A. Van Riper, and J. M. Lattimer
}

We derive the general relativistic Boltzmann equation for massless particles in a stationary axially symmetric metric. The energy and angles of the particles are written in the comoving frame in order to use the local expressions for the collision terms. The zero and first order moment equations in both spectrally integrated and non-integrated form are obtained. From these we derive the thermal equilibrium condition and the diffusion approximation in a relativistic rotating axially symmetric star. We check the equations by recovering the spherically symmetric case and the heat conduction approximation (equilibrium diffusion limit) for axial symmetry. The effects of the rotation on the cooling curves of rotating neutron stars and some observational aspects are studied using the slowly rotating: Hartle [1] formalism together with the cooling equation we derive.

*manuscript in preparation

[1] Hartle, J. B. 1967, Astrophys. J., 150, 1005

\section{Incompressibility of Hot Nuclear Matter, General Relativistic Stellar Col- lapse and Shock Propagation*}

\section{J. A. Miralles, J. M. Ibáñez, J. M. Marti and A. Pérez}

We have performed research on the influence of considering two extreme kinds of Skyrme-type nuclear interactions on the outcome of stellar core collapsing models. Particular attention has been payed to dissociation effects generated by the shock. Neutrino transport is included via a multigroup trapping scheme. When neutrinos are switched off we obtain weak explosions. The explosions are more energetic if we include general relativity and use a soft nuclear force. When neutrino transport is switched on, no explosion is obtained under any conditions.

*Ast. and Astrophys. Supplements, 90, 283 (1991)

\section{Numerical Relativistic Hydrodynamics: A Local Characteristic Approach*}

J. M. Martí, J. M. Ibáñez, and J. A. Miralles

We have extended to the relativistic hydrodynamics system of equations some recent sh sck capturing methods designed for solving nonlinear hyperbolic systems of conservation laws and which avoid the use of artificial viscosity for treating strong discontinuities. Some stanndard shock tube problems and the radial accretion onto a Schwarzschild black hole are use d to calibrate our code.

${ }^{*}$ Phys. Rev. D43, 3794 (1991) 


\section{Ultrarelativistic Hydrodynamics: High Resolution Shock-Capturing Methods*}

A. Marquina (Valencia), J.M. Martí, J.M. Ibáñez, J.A. Miralles and R. Donat (Valencia).

Some high-resolution shock-capturing methods have been designed recently to solve nonlinear hyperbolic systems of conservation laws. We have extended them to the relativistic hydrodynamics system of equation via a local characteristic approach. We are presenting preliminary tests of our procedure in the ultrarelativistic case displaying the main difficulties of this regime and the way to overcome them.

*Astron. Astrophys., 1992 (in press).

\section{Field Theoretical Model for Nuclear and Neutron Matter. V. Slowly Ro- tating Warm Cores in Neutron Stars*}

J.V. Romero (Valencia), J. Diaz Alonso (Paris-Meudon), J.M. Ibáñez, J.A. Miralles and A. Pérez

We have studied the macroscopic properties of general relativistic slowly rotating warm neutron stars obeying the hot extension of an equation of state derived recently in the realm of a field theoretical model. Our results could be of interest in simulations of the early Kelvin epoch of a rotating protoneutron star.

${ }^{*}$ Astrophys. J., 1992 (in press). 

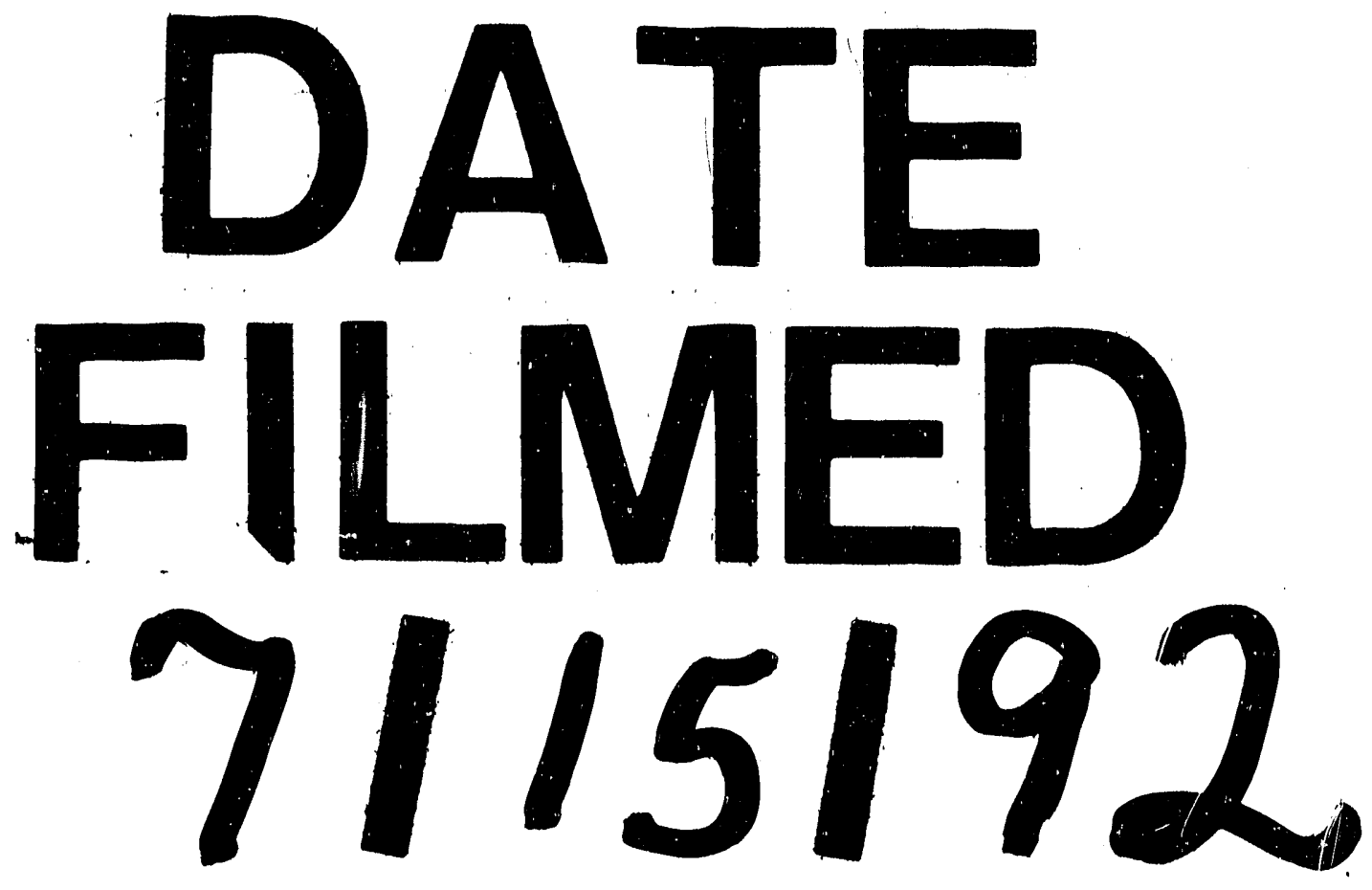
